\title{
Cirurgia minimamente invasiva $x$ cirurgia convencional na taxa de recobrimento radicular e previsibilidade estética: Uma revisão integrativa
}

Minimally invasive surgery $\mathbf{x}$ conventional surgery on rate recovery rate and aesthetic previsibility: An integrative review

Cirugía mínimamente invasiva x cirugía convencional en tasa de recuperación y previsibilidad estética: Una revisión integradora

Silvano Santos Pinheiro

ORCID: https://orcid.org/0000-0002-7093-3610 Universidade Federal do Rio Grande do Norte, Brasil E-mail: sscirurgia@gmail.com

Francisco de Assis de Souza Junior

ORCID: https://orcid.org/0000-0003-3834-2690 Universidade Federal do Rio Grande do Norte, Brasil E-mail: souzajuniorfa@hotmail.com

Isaías Lopes de Medeiros

ORCID: https://orcid.org/0000-0001-8771-0774 Universidade Potiguar, Brasil

E-mail: isaiaslopesm@gmail.com

Bruno Cesar de Vasconcelos Gurgel

ORCID: https://orcid.org/0000-0001-5971-134X Universidade Federal do Rio Grande do Norte, Brasil

E-mail: bcgurgel@yahoo.com.br

Euler Maciel Dantas

ORCID: https://orcid.org/0000-0002-1579-8997 Universidade Federal do Rio Grande do Norte, Brasil E-mail: eulerdantas@yahoo.com.br

Ana Rafaela Luz de Aquino Martins

ORCID: https://orcid.org/0000-0001-7188-4964 Universidade Federal do Rio Grande do Norte, Brasil E-mail: anarafaela.luz@terra.com.br

\begin{abstract}
Resumo
A cirurgia minimamente invasiva (CMI), é um termo que descreve procedimentos cirúrgicos pouco invasivos com ou sem instrumentos de ampliação, porém ainda não existe evidência científica se existe maior previsibilidade estética e de taxa de recobrimento radicular nas CMI em relação a cirurgia para recobrimento radicular convencional. Desta forma, foi realizada uma revisão integrativa da literatura utilizando-se a seguinte estratégia de busca: (microsurgery OR surgical AND minimally invasive surgical OR surgery AND gingival recession AND periodontal plastic surgery OR surgical AND root coverage). nas bases de dados SciELO, PubMed, Scopus, Web of Science, Science Direct, BVS e Google Acadêmico utilizando-se de artigos publicados entre os períodos de 2011 à 2019. No total foram encontrados 546 artigos e após a seleção mediante os critérios de inclusão permaneceram 05 artigos para o estudo, todos ensaios clínicos randomizados. As informações avaliadas dos artigos foram seus objetivos, tamanho da amostra, técnicas cirúrgicas realizadas, o tempo de acompanhamento e os resultados e conclusão. Com base nas análises, podese concluir que a CMI não necessita do uso de instrumentos de magnificação. A tunelização realizada para recobrimento radicular proporciona melhores resultados como ganho tecidual e estético em relação ao Retalho tracionado coronalmente (RTC), principalmente para as classes I e II de Miller. O uso de enxerto conjuntivo associado a CMI aumentou a previsibilidade de resultados satisfatórios.
\end{abstract}

Palavras-chave: Cirurgia minimamente invasiva; Recessão gengival; Cirurgia plástica periodontal.

\begin{abstract}
Minimally invasive surgery (MIS) is a term that describes noninvasive surgical procedures with or without magnification instruments but there is no scientific evidence yet if there is greater aesthetic predictability and root coverage rate in MIS compared to root surgery conventation. Thus, na integrative literatura review was performed using the following searh strategy: (microsurgery OR surgical AND minimally invasive surgical OR surgery AND gingival recession AND periodontal plastic surgery OR surgical AND root coverage). In Scielo, PubMed, Scopus, Web of Science, Science Direct, BVS and Google Scholar databases using articles published between 2011 - 2020. A
\end{abstract}


total of 546 articles were found and after selection according to the inclusion criteria 05 articles remained for the study all randomized clinical trials. The evaluated information of the articles were their objectives sample size surgical techniques performed the follow - up time and the results and conclusion. Based on the analysis it can be concluded that the MIS does not required the use of magnification instuments. Tunneling performed for root coverage provides better results such as tissue and aesthetic gain over the Coronal Traction Flap especially for Miller classes I and II. The use of connective graft associated with MIS increased the predictability of satisfactory results.

Keywords: Minimally invasive surgery; Gingival recession; Periodontal plastic surgery.

\section{Resumen}

La cirugía mínimamente invasiva (MIS) es un término que describe los procedimientos quirúrgicos no invasivos con o sin instrumentos de aumento, pero aún no hay evidencia científica si exi ste una mayor predictibilidad estética y tasa de cobertura radicular en MIS en comparación con la conventación de la cirugía radicular. Por lo tanto, se realizó una revisión integradora de la literatura utilizando la siguiente estrategia de búsqueda: (microsurgery OR surgical AND minimally invasive surgical OR surgery AND gingival recession AND periodontal plastic surgery OR surgical AND root coverage). En las bases de datos Scielo, PubMed, Scopus, Web of Science, Science Direct, BVS y Google Scholar utilizando artículos publicados entre 2011 - 2020. Se encontraron un total de 546 artículos y después de la selección según los criterios de inclusión quedaron 05 artículos para el estudio todos aleatorizados ensayos clínicos. La información evaluada de los artículos fueron sus objetivos, el tamaño de la muestra, las técnicas quirúrgicas realizadas, el tiempo de seguimiento y los resultados y conclusión. Con base en el análisis, se puede concluir que el MIS no requiere el uso de instrumentos de aumento. La tunelización realizada para la cobertura de la raíz proporciona mejores resultados, como ganancia de tejido y estética, sobre el colgajo de tracción coronal, especialmente para las clases Miller I y II. El uso de injerto conectivo asociado con MIS aumentó la previsibilidad de resultados satisfactorios.

Palabras clave: Procedimientos quirúrgicos mínimamente invasivos; Recesión gingival; Cirugía plástica periodontal.

\section{Introdução}

A recessão gengival é resultante de um processo inflamatório decorrente do acúmulo de biofilme dentário ou trauma de escovação. Possui uma prevalência mundial de $88 \%$ em indivíduos com idades superiores a 65 anos e 50\% naqueles com idade entre 18 e 64 anos. (Vieira et al., 2016; Menezes, Kahn, Egreja, Silva \& Machado, 2011) O tratamento das recessões gengivais está incluído nas cirurgias plásticas periodontais e pode ser efetuado mediante técnicas como retalho posicionado coronariamente com ou sem enxerto de tecido conjuntivo subepitelial, retalho posicionado lateralmente e enxerto gengival livre (Kahn, Rodrigues \& Barceleiro, 2013). As abordagens cirúrgicas para os tratamentos podem ser de forma convencional através de cirurgias a retalho ou de maneira minimamente invasiva (Vieira et al., 2016; Menezes et al., 2011; Kahn et al., 2013; Ryder \& Armitage, 2000).

A cirurgia minimamente invasiva (CMI), é um termo que descreve o uso de procedimentos cirúrgicos menores e mais precisos que são possíveis através do uso de instrumentos e materiais microcirúrgicos, com ou sem instrumentos de ampliação, como lupas e microscópios de operação (Cortellini \& Tonetti, 2001; Harrel, Wilson Jr \& Nunn, 2010, Cortellini \& Tonetti, 2005; Ramos et al., 2011; Tunnel \& Harrel, 2017; Harrel et al., 2017, Frizzera et al., 2013; Shruthi, Gujjari \& Mallya, 2015; Bittencourt, Del Peloso Ribeiro, Sallum, Nociti Jr \& Casati, 2012; Chambrone, Pannuti, Tu \& Chambrone, 2012; Cairo, Nieri \& Pagliaro, 2014; Zadeh, 2011; Zuhr, Rebele, Schnieder, Jung \& Hürzeler, 2014; Azaripour et al., 2016; Aimetti, Ferrarotti, Mariani \& Romano, 2017; Chambrone et al., 2010; Kareem, Mahendra \& Kumar, 2018). Possui o objetivo de produzir feridas mínimas, com pouca manipulação tecidual, bem como manuseio suave, seja para tecido duro ou para tecido mole. Enfatiza os aspectos da coagulação e fechamento primário da ferida para a proteção do coágulo sanguíneo. Para a execução das CMIs, a ferida cirúrgica é substancialmente menor que os procedimentos considerados padrões. (Cortellini \& Tonetti, 2001).

Para que um procedimento possa ser considerado como minimamente invasivo, ele não precisa, ser realizado com aparelhos de magnificação, muito embora o uso de microscópio cirúrgico ou de lupa facilite a visualização da área de interesse, reduzindo o tamanho das incisões e o dano tecidual (Harrel et al., 2010). Os autores Cortellini e Tonetti (2005), sugerem que a CMI resulta em um potencial melhorado de fechamento dos casos obtidos com cirurgia regular. 
Na periodontia, procedimentos minimamente invasivos foram descritos primeiramente por Harrel em 1995, o qual relatou uma técnica de menor invasividade para remoção de tecido de granulação e enxerto ósseo (Ramos et al., 2011). Conforme a Academia Americana de Periodontologia (1996), a CMI, vem despontando como uma forma de refinamento das técnicas cirúrgicas existentes sendo uma nova abordagem para conseguir a regeneração periodontal sem as desvantagens associadas à cirurgia convencional de retalho.

Porém na literatura ainda não está definido se a CMI nos procedimentos periodontais produz melhores resultados que as cirurgias de cunho convencional para o recobrimento de recessões gengivais. Mediante ao exposto este trabalho propõe-se responder à pergunta: Existe maior previsibilidade estética e de taxa de recobrimento radicular nas cirurgias minimamente invasivas em relação a cirurgia convencional?

\section{Metodologia}

Os artigos científicos foram obtidos através da busca online nas bases de dados SciELO (Scientific Eletronic Library On line), PubMed / MEDLINE; SCOPUS. (Public Medicine), Web of Science, Science Direct, BVS (Biblioteca Virtual em Saúde) e Google Acadêmico para identificar se existe maior previsibilidade estética e de taxa de recobrimento radicular nas cirurgias minimamente invasivas em relação a cirurgia convencional. A estratégia de busca consistiu na combinação dos seguintes descritores: (microsurgery OR surgical) AND (minimally invasive surgical OR minimally invasive surgery) AND (gingival recession) AND (periodontal plastic surgery OR periodontal plastic surgical) AND (root coverage). Vale salientar que não houve alterações dos descritores em nomenclatura nem em forma disposta da diferente acima relatada.

Foram incluídos ensaios clínicos randomizados, desde que realizassem comparações entre as técnicas minimamente invasiva e convencionais para recobrimento radicular, publicados entre janeiro de 2011 a outubro de 2018, nos idiomas português, inglês e espanhol. Inicialmente, foram selecionados os títulos e resumos que continham os descritores ou sugeriam alguma relação com o objetivo do trabalho. Em seguida, somente foram lidos os artigos completos que preenchiam os critérios de inclusão. Além disso, foi realizada busca manual em revistas mais relevantes da área da periodontia, como também, a partir da bibliografia dos artigos selecionados.

A busca foi realizada por 2 pesquisadores de forma independente onde apenas as discordâncias foram analisadas em conjunto. Informações dos estudos foram coletadas com ênfase nos seguintes dados: autores, ano de publicação, objetivos do estudo, características metodológicas dos estudos, população de estudo - quanto à idade e ao sexo, tipo de técnica cirúrgica utilizada, tipo de recessão radicular tratada de acordo com a classificação de Miller, tempo de acompanhamento, resultados e conclusões dos estudos.

\section{Resultados e Discussão}

$\mathrm{Na}$ primeira busca foram encontrados um total de 546 artigos no total, sendo que destes, 442 apresentavam-se duplicados nas bases de dados. Após a leitura de título e resumo das 100 publicações previamente selecionadas, foram excluídos 80 estudos por não atenderem aos critérios de inclusão. 20 artigos que abordavam o tema proposto foram lidos na íntegra. Após esta leitura 10 foram excluídos por não apresentaram comparação entre as técnicas e 7 não possuíam uma metodologia adequada ao estudo, sendo assim, permaneceram apenas 3 estudos. Figura 1. Os artigos incluídos e analisados na pesquisa foram descritos na Tabela 1 . 
Research, Society and Development, v. 10, n. 5, e17610514314, 2021

(CC BY 4.0) | ISSN 2525-3409 | DOI: http://dx.doi.org/10.33448/rsd-v10i5.14314

Figura 1: Fluxograma da seleção dos artigos.

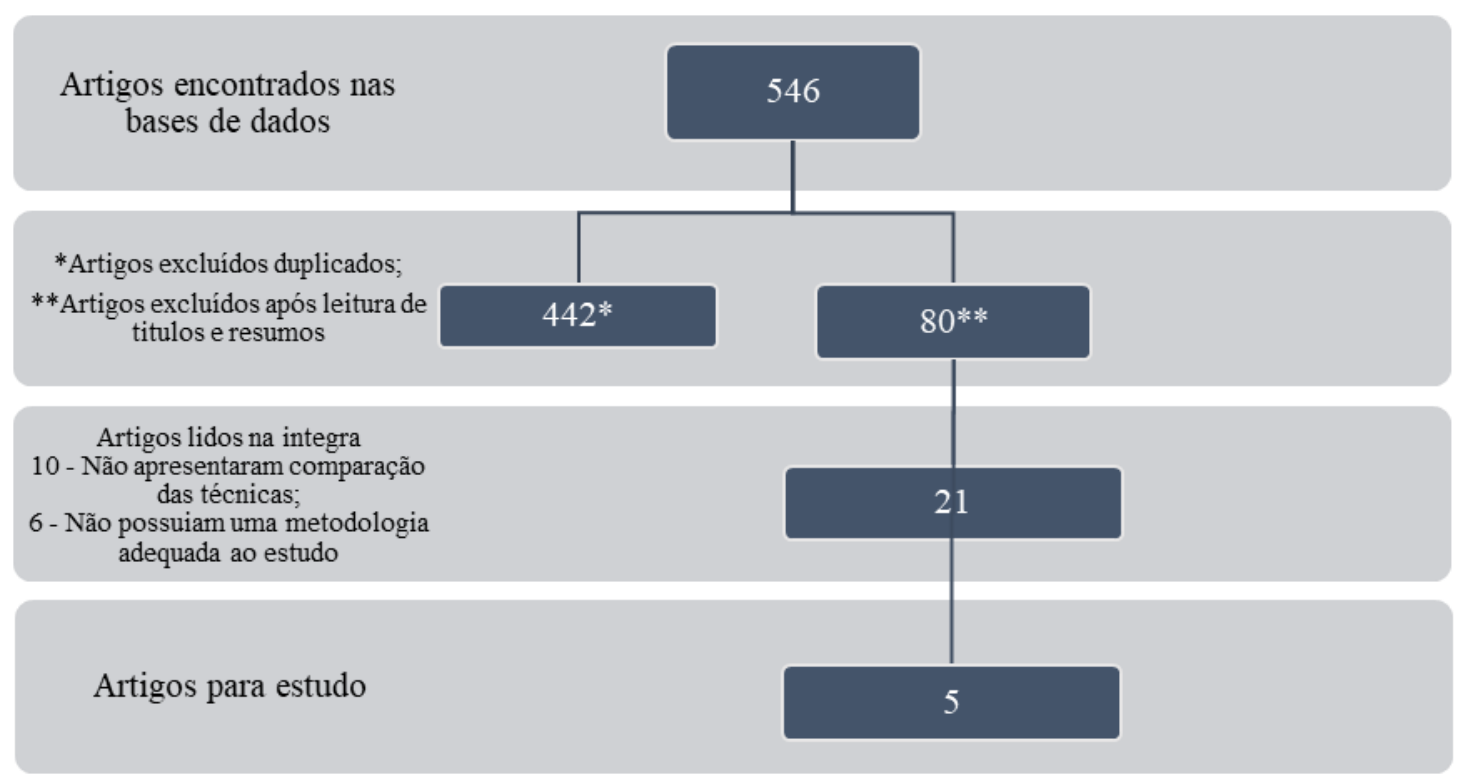

Fonte: Autores. 
Tabela 1 - Análise dos artigos incluídos na pesquisa.

\begin{tabular}{|c|c|c|c|c|c|c|c|c|}
\hline Autores (Ano) & $\begin{array}{l}\text { Objetivos } \\
\text { do estudo }\end{array}$ & $\begin{array}{l}\text { Caracte } \\
\text { rísticas } \\
\text { metodol } \\
\text { ógicas }\end{array}$ & $\begin{array}{l}\text { População de } \\
\text { estudo idade } \\
\text { e sexo }\end{array}$ & $\begin{array}{l}\text { Técnica } \\
\text { cirúrgica } \\
\text { utilizada }\end{array}$ & $\begin{array}{c}\text { Recessão } \\
\text { radicular tratada } \\
\text { (classificação de } \\
\text { Miller) }\end{array}$ & $\begin{array}{c}\text { Tempo de } \\
\text { acompanha } \\
\text { mento }\end{array}$ & Resultados & Conclusões \\
\hline $\begin{array}{l}\text { BITTENCOURT } \\
\text { et. al. (2012) }\end{array}$ & $\begin{array}{l}\text { Comparar a } \\
\text { cobertura } \\
\text { radicular, a } \\
\text { morbidade } \\
\text { pós-operatória } \\
\text { e os resultados } \\
\text { estéticos da } \\
\text { técnica de } \\
\text { SCTG com ou } \\
\text { sem o uso de } \\
\text { microscópio } \\
\text { cirúrgico no } \\
\text { tratamento de } \\
\text { recessões } \\
\text { gengivais. }\end{array}$ & ECR & $\begin{array}{l}24 \text { pacientes ( } 13 \\
\text { masculino e } 11 \\
\text { feminino) entre } \\
18 \text { e } 55 \text { anos }\end{array}$ & $\begin{array}{l}\text { Grupo teste } \\
\text { (G1) SCTG } \\
\text { realizada } \\
\text { com um } \\
\text { microscópio. } \\
\text { Grupo } \\
\text { controle (G2) } \\
\text { SCTG foi } \\
\text { realizada sem } \\
\text { microscópio. }\end{array}$ & $\begin{array}{l}\text { Classe I ou II em } \\
\text { Caninos ou pré- } \\
\text { molares superiores, } \\
\text { sendo } 26 \text { caninos e } \\
22 \text { pré molares. }\end{array}$ & $\begin{array}{l}12 \text { meses pós } \\
\text { operatórios } \\
\text { por um único } \\
\text { pesquisador }\end{array}$ & $\begin{array}{l}\text { Numa avaliação intra grupo foram } \\
\text { encontrados diferenças estatísticas } \\
(\mathrm{P}<0,05) \text {, após } 12 \text { meses, aumento nos } \\
\text { parâmetros WKT, ETQ e PD. } \\
\text { Diminuição em RL, NLC e AR. } \\
\text { Avaliação intergrupos os valores mais } \\
\text { elevados de RA no grupo controle. } 12 \\
\text { meses após a cirurgia, a porcentagem } \\
\text { de cobertura foi de } 98,0 \% \text { e } 88,3 \% \text { para } \\
\text { teste e controle, respectivamente } \\
\text { (P <0,05) Após } 12 \text { meses, a cobertura } \\
\text { radicular completa foi atingida em } \\
87,5 \% \text { (21 de } 24) \text { dos casos tratados no } \\
\text { grupo de teste e em } 58,3 \% \text { (14 de } 24) \\
\text { no grupo de controlo. }\end{array}$ & $\begin{array}{l}\text { Ambas as abordagens são } \\
\text { capazes de produzir } \\
\text { cobertura radicular, } \\
\text { entretanto, o uso do } \\
\text { microscópio cirúrgico é } \\
\text { associadas a benefícios } \\
\text { clínicos adicionais no } \\
\text { tratamento } \\
\text { de dentes com recessões } \\
\text { gengivais. }\end{array}$ \\
\hline ZUHR (2013) & $\begin{array}{l}\text { Introduzir } \\
\text { métodos de } \\
\text { medição } \\
\text { digital 3D } \\
\text { para avaliar os } \\
\text { resultados } \\
\text { após a CR } \\
\text { cirurgica e } \\
\text { avaliar o } \\
\text { desempenho } \\
\text { clínico da } \\
\text { técnica do } \\
\text { túnel com } \\
\text { enxerto de } \\
\text { TUN e CAF } \\
\text { com derivado } \\
\text { de matriz de } \\
\text { esmalte no } \\
\text { tratamento de } \\
\text { defeitos de }\end{array}$ & ECR & $\begin{array}{l}24 \text { pacientes ( } 17 \\
\text { feminino e } 7 \\
\text { masculino), } \\
\text { média de idade } \\
38 \text { anos }\end{array}$ & $\begin{array}{l}\text { TUN + CTG } \\
\text { vs CAF + } \\
\text { EMD }\end{array}$ & Classe Miller I e II & $\begin{array}{l}\text { Avaliação } \\
\text { entre } 6 \text { e } 12 \\
\text { meses }\end{array}$ & $\begin{array}{l}\text { O seguimento de } 6 \text { meses, o CR foi } \\
99,2 \pm 1,6 \% \text { para o grupo TUN e } 72,2 \pm \\
18,4 \% \text { para o grupo CAF }(\mathrm{p}=0,0001) \text {. } \\
\text { A CRC foi detectado em } 80,0 \% \text { dos } \\
\text { sítios tratados com TUN e em } 15,4 \% \\
\text { dos sítios tratados com CAF ( } \mathrm{p}= \\
0,0020) \text {. } \\
\text { No seguimento de } 12 \text { meses, o CR foi, } \\
\text { em média, de } 98,4 \pm 3,6 \% \text { para o grupo } \\
\text { TUN e de } 71,8 \pm 20,3 \% \text { para o grupo } \\
\text { CAF ( }=0,0004) \text {. } \\
\text { A redução de AR foi de } 1,94 \times 0,57 \mathrm{~mm} \\
\text { em locais tratados com TUN ( } 0,04 \pm \\
0,07 \text { mm após } 12 \text { meses), enquanto que } \\
1,17 \quad \pm \quad 0,42 \text { mm de AR } \\
\text { (Correspondente a um resíduo médio de } \\
0,550,49 \text { mm) foi registrada no grupo } \\
\text { CAF ( } \mathrm{p}=0,0015) \text {. } \\
\text { A CRC foi detectado em } 11 \text { de } 14\end{array}$ & $\begin{array}{l}\text { TUN resultou em melhores } \\
\text { resultados clínicos } \\
\text { juntamente com melhores } \\
\text { resultados finais em relação } \\
\text { ao CAF } \\
\text { O TUN oferece uma } \\
\text { elegante opção de } \\
\text { tratamento, especialmente } \\
\text { em casos de multipla } \\
\text { recessão; } \\
\text { O novo método de medição } \\
\text { ofereceu alta precisão. }\end{array}$ \\
\hline
\end{tabular}


Research, Society and Development, v. 10, n. 5, e17610514314, 2021

(CC BY 4.0) | ISSN 2525-3409 | DOI: http://dx.doi.org/10.33448/rsd-v10i5.14314

\begin{tabular}{|c|c|c|c|c|c|c|c|c|}
\hline & $\begin{array}{l}\text { recessão } \\
\text { gengival } \\
\text { localizados } \\
\text { superficiais. }\end{array}$ & & & & & & $\begin{array}{l}\text { locais }(78,6 \%) \text { analisados em } 12 \text { meses } \\
\text { no grupo TUN e em } 3 \text { de } 14(21,4 \%) \text { de } \\
\text { locais tratados com CAF }(\mathrm{p}=0,0070) \text {. } \\
\text { No que diz respeito ao HKT, as } \\
\text { diferenças no Mudança de altura de } \\
\text { queratina entre os dois grupos foram } \\
\text { estatisticamente significativas após } 6 \text { (p } \\
=0,0009) \text {. }\end{array}$ & \\
\hline $\begin{array}{l}\text { AZARIPOUR } \\
\text { et.al. (2016) }\end{array}$ & $\begin{array}{l}\text { comparar o } \\
\text { CAF com a } \\
\text { TTMM para o } \\
\text { tratamento de } \\
\text { recessões } \\
\text { Miller classe I } \\
\text { e II. }\end{array}$ & $\begin{array}{l}\text { ECR } \\
\text { duplo } \\
\text { cego }\end{array}$ & $\begin{array}{l}40 \text { pacientes }(25 \\
\text { femininos e } 15 \\
\text { masculinos), } \\
\text { com idade } \\
\text { média de } 39 \\
\text { anos. }\end{array}$ & $\begin{array}{l}\text { CAF vs } \\
\text { TTMM }\end{array}$ & Classe I e II. & $\begin{array}{l}\text { Avaliações } \\
\text { entre } 6 \text { e } 12 \\
\text { meses. }\end{array}$ & 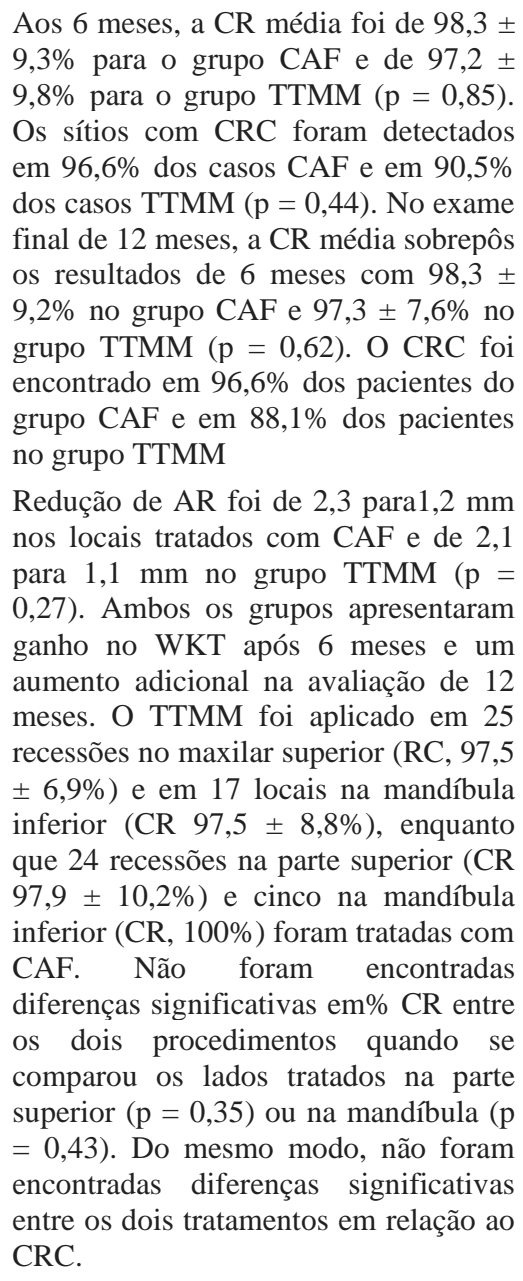 & $\begin{array}{l}\text { CAF e TTMM com o uso } \\
\text { adicional de enxerto são } \\
\text { igualmente bem sucedidos } \\
\text { na cobertura das recessões } \\
\text { gengivais de classe Miller I } \\
\text { e II, com altos resultados } \\
\text { estéticos. Todos os } \\
\text { pacientes indicaram sua } \\
\text { disposição para a cirurgia } \\
\text { periodontal adicional. }\end{array}$ \\
\hline
\end{tabular}


Research, Society and Development, v. 10, n. 5, e17610514314, 2021

(CC BY 4.0) | ISSN 2525-3409 | DOI: http://dx.doi.org/10.33448/rsd-v10i5.14314

\begin{tabular}{|c|c|c|c|c|c|c|c|c|}
\hline $\begin{array}{l}\text { RIBEIRO, F.V. } \\
\text { et. al. (2013) }\end{array}$ & $\begin{array}{l}\text { Comparar } \\
\text { desfecho } \\
\text { clínico de } \\
\text { recobrimento } \\
\text { radicular pela } \\
\text { técnica de } \\
\text { retalho aberto } \\
\text { e flapless. }\end{array}$ & $\begin{array}{l}\text { Estudo } \\
\text { randomiz } \\
\text { ado } \\
\text { controlad } \\
\text { o com } \\
\text { boca } \\
\text { dividida }\end{array}$ & $\begin{array}{l}28 \text { pacientes }(8 \\
\text { homens e } 20 \\
\text { mulheres) }\end{array}$ & $\begin{array}{l}\text { Cirurgia } \\
\text { aberta } \mathrm{x} \\
\text { minimamente } \\
\text { invasiva }\end{array}$ & $\begin{array}{l}\text { Defeitos estéticos } \\
\text { gengivais (aumento } \\
\text { de coroa) }\end{array}$ & 12 meses & $\begin{array}{l}\text { Os pacientes não relataram efeitos } \\
\text { adversos. O tempo cirúrgico médio foi } \\
\text { menor na cirurgia flapless (média } 12-31 \\
\text { min) do que na cirurgia aberta (média } \\
\text { 14-41min), variação } 20 \text { a } 66 \text { min } \\
\text { (estatisticamente significativa) }\end{array}$ & $\begin{array}{l}\text { Ambas as modalidades } \\
\text { terapêuticas produziram } \\
\text { resultados similares após } 12 \\
\text { meses. }\end{array}$ \\
\hline
\end{tabular}

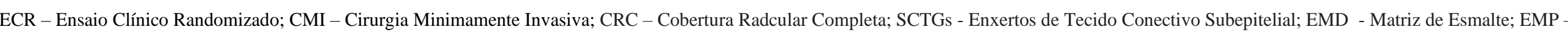

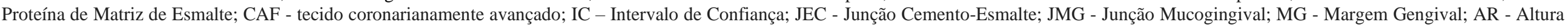

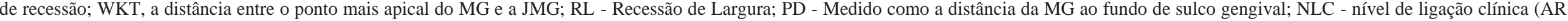

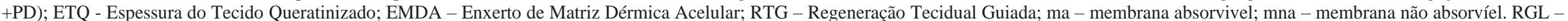

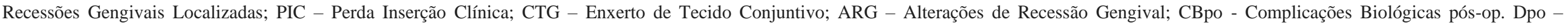

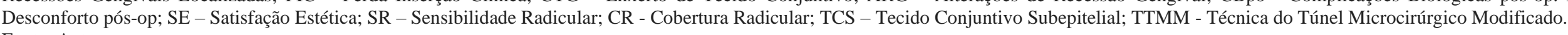
Fonte: Autores. 


\section{Discussão}

O presente estudo se propôs a avaliar, mediante uma revisão integrativa, se existe maior previsibilidade estética e de taxa de recobrimento radicular nas cirurgias minimamente invasivas em relação a cirurgia convencional. Todos os artigos analisados consideraram como CMI, os procedimentos conservadores que visaram minimizar o traumatismo tecidual resultando na manutenção do suprimento sangüíneo, na estabilidade do coágulo no local da ferida e na diminuição da recessão pós-operatória ao longo do tempo.

Então, apesar de muitos estudos (Tunnel \& Harrel, 2017; Harrel et al., 2017; Frizzera et al., 2013; Shruthi et al., 2015; Bittencourt et al., 2012) referirem-se a CMI com o uso de aparelhos de magnificação, este é uma forma auxiliar para realizar a CMI. Dentre as vantagens citadas com a utilização desses dispositivos podem-se destacar uma melhor acuidade visual e um bom posicionamento ergonômico, porém sem efeitos significativos quanto ao resultado final da CMI (Bittencourt et al., 2012; Chambrone et al., 2012; Cairo et al., 2014; Zadeh, 2011; Zuhr et al., 2014; Azaripour et al., 2016; Aimetti et al., 2017; Chambrone et al., 2010; Kareem et al., 2018).

Algumas técnicas cirúrgicas para recobrimento radicular como a tunelização, são consideradas por natureza como procedimentos minimamente invasivos (Zadeh, 2011). Para Zuhr et al. (2014) a tunelização oferece uma excelente opção de tratamento, especialmente em casos de multipla recessão. No seu estudo o qual comparou a técnica de tunelização (TUN) com o retalho tracionado coronalmente (RTC) pode-se perceber que após 6 meses de acompanhamento, o recobrimento radicular foi de $99,2 \%$ para o grupo de TUN e 72,2\% para o grupo de RTC. Essa diferença significativa também se manteve após 12 meses da cirurgia $(p<0,01)$. Dentre os parâmetros avaliados nesse estudo a altura de recessão (AR) foi dimunída consideravelmente na técnica de TUN chegando em alguns pontos avaliados a ser de 1,94mm e a do RTC foi no máximo de 1,17mm de diminuição, chegando a ter resíduos de recessão após 12 meses nessa técnica.

Ainda nesse estudo, a cobertura radicular completa foi de 78,7\% após 12 meses com a técnica de tunelização e a do tecido avançado coronalmente foi de 21,4\%. Esteticamente as técnicas cirúrgicas apresentaram resultados favoráveis.

Resultados satisfatórios para a tunelização também foram encontrados no estudo de Azaripour et al. (2016) em que eles compararam as duas técnicas TUN e RTC. Ambas utilizaram enxertos de tecido conjuntivo. Numa avaliação após 6 meses de cirurgia, a cobertura radicular foi de $98,3 \%$ para a técnica de RTC e 97,2\% para a técnica de TUN apesar de não ser estatisticamente significante a diferença ( $p>0,05)$. Após 12 meses os mesmos valores foram mantidos. A altura de recessão foi diminuída no RTC em média 1,1mm e 1,0mm para a TUN. Em ambos os grupos houve ganho significativo de queratina após 6 meses. Pode-se constatar que ambas as técnicas foram bem-sucedidas tanto em ganho tecidual, como também, em resultados estéticos.

Uma diferença do estudo de Zuhr et al. (2014) é que apesar de comparar as duas técnicas TUN e RTC, os enxertos utilizados nessas técnicas foram diferentes, sendo enxerto de matriz de esmalte para TUN e enxerto de tecido conjuntivo para RTC, isso pode explicar a diferença estatística entre as técnicas.

Para Aimetti et al. (2017), as técnicas minimamente invasivas (uso de ampliação e instrumentais microcirúrgicos) com a finalidade de recobrimento radicular promovem excelentes resultados. Em seu estudo realizado com 30 pacientes, comparando técnicas de recobrimento radicular sem retalho versus técnica minimamente invasiva sem o uso de microscópio, ambas utilizando matriz de esmalte como enxerto proporcionaram resultados satisfatórios nos parâmetros analisados como profundidade de sondagem e ganho de inserção clínica.

O mesmo autor, numa avaliação de 24 meses pós cirurgia, os locais tratados sem retalho obtiveram uma redução de profundidade em média de $3,6 \mathrm{~mm}$ e ganho de inserção clínica em torno de 3,2 $\mathrm{mm}$. Para o grupo tratado com procedimento minimamente invasivo a profundidade de sondagem teve redução em média de 3,7mm e ganho de inserção clínica de 3,6mm. Todos os ensaios clínicos incluídos nesse estudo trataram de recessão classe I e II de Miller. Então, mediante ao exposto, pode 
ser considerado que a cirurgia minimante invasiva fornece melhores resultados quando comparada com cirurgias ditas convencionais. Vale ressaltar que todos os estudos analisados com a técnica minimamente invasiva não mencionaram dores pós-operatórias significantes. E o estudo de Aimetti et al (2017) além dessa consideração frisou o ganhou de inserção clínica para ambos os procedimentos minimamente invasivos analisados por eles, como também faz menção que na cirurgia plástica periodontal, a escolha do procedimento baseia-se nos princípios de sucesso, reprodutibilidade da dor operatória.

\section{Conclusão}

Mediante ao exposto no presente estudo é possível inferir que a CMI não necessita do uso de instrumentos de magnificação como lupas e microscópios, porém estes proporcionam uma melhor acuidade visual, melhor iluminação do campo operatório e sugere melhora no posicionamento ergonômico.

Das técnicas de CMI avaliadas, a tunelização realizada para recobrimento radicular proporciona excelentes resultados como ganho tecidual e estético em relação ao RTC, tendo aumento real maior que $1 \mathrm{~mm}$ principalmente para as classes I e II de Miller, sendo avaliados após 6 meses e os resultados foram mantidos após 1 ano.

Os enxertos associados com a CMI também favoreceram para o ganho de inserção, sendo o enxerto de tecido conjuntivo com resultado mais satisfatório em relação ao enxerto de matriz do esmalte.

\section{Referências}

Aimetti, M., Ferrarotti, F., Mariani, G. M., \& Romano, F. (2017). A novel flapless approach versus minimally invasive surgery in periodontal regeneration with enamel matrix derivative proteins: a 24-month randomized controlled clinical trial. Clinical oral investigations, 21 (1), 327-337.

Azaripour, A., Kissinger, M., Farina, V. S. L., Van Noorden, C. J., Gerhold-Ay, A., Willershausen, B., \& Cortellini, P. (2016). Root coverage with connective tissue graft associated with coronally advanced flap or tunnel technique: a randomized, double-blind, mono-centre clinical trial. Journal of clinical periodontology, 43(12), 1142-1150.

Bittencourt, S., Del Peloso Ribeiro, É., Sallum, E. A., Nociti Jr, F. H., \& Casati, M. Z. (2012). Surgical microscope may enhance root coverage with subepithelial connective tissue graft: A randomized-controlled clinical trial. Journal of periodontology, 83(6), 721-730.

Cairo, F., Nieri, M., \& Pagliaro, U. (2014). Efficacy of periodontal plastic surgery procedures in the treatment of localized facial gingival recessions. A systematic review. Journal of clinical periodontology, 41, S44-S62.

Chambrone, L., Pannuti, C. M., Tu, Y. K., \& Chambrone, L. A. (2012). Evidence-based periodontal plastic surgery. II. An individual data meta-analysis for evaluating factors in achieving complete root coverage. Journal of periodontology, 83(4), 477-490.

Chambrone, L., Sukekava, F., Araújo, M. G., Pustiglioni, F. E., Chambrone, L. A., \& Lima, L. A. (2010). Root-coverage procedures for the treatment of localized recession-type defects: A Cochrane systematic review. Journal of periodontology, 81(4), 452-478.

Cortellini, P., \& Tonetti, M. S. (2001). Microsurgical approach to periodontal regeneration. Initial evaluation in a case cohort. Journal of Periodontology, 72(4), 559-569.

Cortellini, P., \& Tonetti, M. S. (2005). Clinical performance of a regenerative strategy for intrabony defects: scientific evidence and clinical experience. Journal of periodontology, 76(3), 341-350.

Frizzera, F., Vieira, G. H., Molon, R. S., Peçanha, M. M., Marcantonio Jr, E., \& Sampaio, J. E. C. (2013). Recobrimento radicular para o tratamento de hipersensibilidade dentinária cervical persistente. Perionews, 42-49.

Harrel, S. K., Nunn, M. E., Abraham, C. M., Rivera-Hidalgo, F., Shulman, J. D., \& Tunnell, J. C. (2017). Videoscope assisted minimally invasive surgery (VMIS): 36-month results. Journal of periodontology, 88(6), 528-535.

Harrel, S. K., Wilson Jr, T. G., \& Nunn, M. E. (2010). Prospective assessment of the use of enamel matrix derivative with minimally invasive surgery: 6-year results. Journal of periodontology, 81(3), 435-441.

Kahn, S., Rodrigues, W. J. D. P. R., \& Barceleiro, M. D. O. (2013). Periodontal plastic microsurgery in the treatment of deep gingival recession after orthodontic movement. Case reports in dentistry, 2013.

Kareem, N., Mahendra, J., \& Kumar, K. A. (2018). Triangular coronally advanced flap: Conventional versus Microsurgery. Journal of Indian Society of Periodontology, 22(1), 73.

Menezes, C. C. D., Kahn, S., Egreja, A. M. C., Silva, J. A. D., \& Machado, W. A. (2011). Periodontal microsurgery: a Brazilian overview. RGO. Revista Gaúcha de Odontologia (Online), 59(4), 583-589.

Ramos, U., Novaes Jr, A., Souza, S., Palioto, D., Taba Jr, M. \& Grisi, M., 2011. Minimally invasive procedures in periodontology and implantology. Braz J Periodontol, 21(4), pp.32-38. 
Research, Society and Development, v. 10, n. 5, e17610514314, 2021

(CC BY 4.0) | ISSN 2525-3409 | DOI: http://dx.doi.org/10.33448/rsd-v10i5.14314

Ryder, M. I., \& Armitage, G. C. (2016). Minimally invasive periodontal therapy for general practitioners. Periodontology 2000, 71(1), 7-9.

Shruthi, S., Gujjari, S. K., \& Mallya, K. P. (2015). Comparison of two surgical techniques for the reconstruction of interdental papilla. Journal of Interdisciplinary Dentistry, 5(1), 17.

Tunnell, J. C., \& Harrel, S. K. (2017). Minimally Invasive Surgery in Periodontal Regeneration: A Review of the Literature. Compendium of continuing education in dentistry (Jamesburg, NJ: 1995), 38(4), e13-e16.

Vieira, T. R., de Oliveira, D. W. D., Lages, F. S., Soares, R. V., Horta, M. C. R., \& Zenóbio, E. G. (2016). Effect of Periodontal Parameters on Root Coverage. Journal of the International Academy of Periodontology, 18(3), 86-93.

Zadeh, H. H. (2011). Minimally invasive treatment of maxillary anterior gingival recession defects by vestibular incision subperiosteal tunnel access and platelet-derived growth factor BB. International Journal of Periodontics and Restorative Dentistry, 31(6), 653.

Zuhr, O., Rebele, S. F., Schneider, D., Jung, R. E., \& Hürzeler, M. B. (2014). Tunnel technique with connective tissue graft versus coronally advanced flap with enamel matrix derivative for root coverage: a RCT using 3D digital measuring methods. Part I. Clinical and patient-centred outcomes. Journal of clinical periodontology, 41(6), 582-592. 